\title{
Chiral-anomaly-induced angular narrowing of the positive longitudinal magnetoconductivity in Weyl semimetals
}

\author{
Ming-Xun Deng $\odot,{ }^{1}$ Jia-Yan Ba, ${ }^{1}$ R. Ma,${ }^{2}$ Wei Luo $\odot,{ }^{3}$ Rui-Qiang Wang, ${ }^{1,}$ L. Sheng,${ }^{4,5, \dagger}$ and D. Y. Xing ${ }^{4,5}$ \\ ${ }^{1}$ Guangdong Provincial Key Laboratory of Quantum Engineering and Quantum Materials, \\ GPETR Center for Quantum Precision Measurement, SPTE, South China Normal University, Guangzhou 510006, China \\ ${ }^{2}$ Jiangsu Key Laboratory for Optoelectronic Detection of Atmosphere and Ocean, \\ Nanjing University of Information Science and Technology, Nanjing 210044, China \\ ${ }^{3}$ School of Science, Jiangxi University of Science and Technology, Ganzhou 341000, China \\ ${ }^{4}$ National Laboratory of Solid State Microstructures and Department of Physics, Nanjing University, Nanjing 210093, China \\ ${ }^{5}$ Collaborative Innovation Center of Advanced Microstructures, Nanjing University, Nanjing 210093, China
}

(Received 17 May 2020; revised 18 July 2020; accepted 5 August 2020; published 1 September 2020)

\begin{abstract}
By generalizing the Landau quantization Boltzmann equation to a finite-size system, we investigate the magnetotransport in disordered Weyl semimetals (WSMs) for both the ballistic and diffusive regimes. It is found that, in the diffusive limit, the chiral chemical potential would drive extra electrical current to flow through the chiral channels in the $n=0$ Landau levels, leading to emergence of the positive longitudinal magnetoconductivity (LMC). The positive LMC will exhibit the angular narrowing phenomenon if $\hbar \omega_{c} \gg$ $E_{\mathrm{F}} \sqrt{l_{\mathrm{a}} / l_{\mathrm{e}}}$, where $\omega_{c}, E_{\mathrm{F}}$, and $l_{\mathrm{a}(\mathrm{e})}$ denote, respectively, the cyclotron frequency, the Fermi energy, and the intravalley (intervalley) relaxation length. The anomalous magnetic field dependence of the angular narrowing, i.e., the width and height of the positive LMC peak could increase or decrease with increasing the magnetic field, can be attributable to quantum oscillations of the chiral anomaly. In addition, the quantum oscillations are sensitive to the temperature and impurity scattering, which may also cause some other anomalous properties for the LMC. Our findings are helpful to understand the anomalous behaviors of the positive LMC in WSMs.
\end{abstract}

DOI: 10.1103/PhysRevResearch.2.033346

\section{INTRODUCTION}

The recent theoretical and experimental discovery of Dirac and Weyl semimetals (WSMs) provides the possibility for realization of the physics of relativistic fermions in solid-state physics [1-11]. The low-energy electronic excitations of a WSM behave as massless Weyl quasiparticles, and their dispersions form a nondegenerate three-dimensional cone around an isolated band-touching point, called the Weyl node. The Weyl nodes are monopoles of the Berry curvature, which always come in pairs with opposite chiralities in momentum space [12,13], and a pair of Weyl nodes are connected by the open Fermi-arc surface states. The ultrahigh mobility and spectacular transport properties of the charged Weyl fermions can find applications in high-speed electronic circuits and computers [14-16]. Motivated both by the interesting physics and potential applications, WSMs have recently spurred intensive and innovative research in the field of condensed-matter physics [17-24].

\footnotetext{
*wangruiqiang@m.scnu.edu.cn

${ }^{\dagger}$ shengli@nju.edu.cn
}

Published by the American Physical Society under the terms of the Creative Commons Attribution 4.0 International license. Further distribution of this work must maintain attribution to the author(s) and the published article's title, journal citation, and DOI.
The topologically protected Weyl nodes and Fermi-arc surface states, which can be observed directly by the angleresolved photoelectron spectroscopy (ARPES), are regarded as the most distinctive observable spectroscopic feature of WSMs $[1,8-10,25]$. However, the ARPES observation is sometimes limited by the spectroscopic resolutions, especially for disordered WSMs, whose spectrum and Weyl nodes could be obscured by the impurity scattering [26]. Therefore, it is urgent to find alternative physical characteristics as a smoking gun for identifying WSMs. Of particular interest is the very unusual positive longitudinal magnetoconductivity (LMC) or negative magnetoresistivity resulting from the chiral anomaly of the Weyl fermions [27-30]. While the positive LMC was observed experimentally in a variety of WSM materials [31-38], some of its measured dependence on the angle $\theta$ between the electric and magnetic fields [31,37] appeared to be in contradiction with the theoretical expectations [27-29,39]. In most situations, it is predicted that the chiral-anomaly-induced LMC will follow the $\cos ^{2} \theta$ dependence, whose amplitude is linearly or quadratically dependent on $B$, where $B$ is the strength of the magnetic field. However, some experiments, e.g., as indicated by Figs. 3(c)-3(e) of Ref. [37], showed that the positive LMC could exhibit a quite narrow angular dependence around $\theta=0$. Moreover, with increasing the magnetic field, the LMC peak could reduce, even by an order in magnitude, as compared in Figs. 3(d) and 3(e) of Ref. [37]. In the diffusive and low magnetic field limits, Burkov et al. presented a possible explanation for the 
angular narrowing phenomenon [40,41], but the anomalous magnetic field dependence of the LMC peak deserves further research. Furthermore, with increasing the magnetic field, the LMC would experience a crossover from the classical to ultraquantum limit, where the LMC will exhibit interesting quantum oscillations $[30,42,43]$. It is highly desirable to explore the interplay between the quantum oscillations and angular dependence of the LMC.

In this paper, by generalizing the Boltzmann equation incorporating Landau quantization to a finite-size system, we study the chiral anomaly of disordered WSMs in both the ballistic and diffusive limits. We derive an analytical formula for the chiral-anomaly-induced positive LMC, which is applicable for the parameters ranging from the classical to ultraquantum limit. It is found that in the diffusive regime, the nonequilibrium chiral chemical potential due to chiral anomaly will drive extra electrical current to flow through the chiral channels in the $n=0$ Landau levels (LLs). The resulting positive LMC could exhibit the angular narrowing effect, if $\hbar \omega_{c} \gg E_{\mathrm{F}} \sqrt{l_{\mathrm{a}} / l_{\mathrm{e}}}$, where $\omega_{c}$ denotes the cyclotron frequency, $E_{\mathrm{F}}$ stands for the Fermi energy, and $l_{\mathrm{a}(\mathrm{e})}$ represents the intravalley (intervalley) relaxation length. As a result, the properties of the angular narrowing, in addition to the relaxation lengths proposed in Ref. [41], are tunable by the relative magnitudes of the Fermi energy and magnetic field. Moreover, the angular narrowing of the LMC can display anomalous behaviors associated with the quantum oscillations. For a fixed magnetic field strength, the LMC can increase or decrease with increasing temperature or strength of the impurity scattering, depending on whether the field strength is near a valley or near a peak of the energy spectrum of the LLs, while, for a fixed Fermi energy, if increasing the magnetic field pushes a valley to the Fermi level, the height of the LMC peak would decrease, drastically. Our findings are helpful to understand the observed angular narrowing of the LMC in WSMs. The rest of this paper is organized as follows. In Sec. II, we introduce the model and theory and present the results and discussions in Sec. III. The last section contains a summary.

\section{MODEL AND THEORY}

Let us start by considering an experimental setup, in which a WSM of length $L_{z}$ is attached to current-carrying normal metal (NM) electrodes at $z=0$ and $L_{z}$, as shown in Fig. 1. For simplicity, the widths of the system in the $x$ and $y$ directions are assumed to be infinite, equivalent to the periodic boundary condition, which would not influence the qualitative results. The low-energy excitation of the WSM with two Weyl nodes in a magnetic field can be described by an effective continuum Hamiltonian

$$
H_{\chi}(\mathbf{k})=\chi \hbar v_{\mathrm{F}} \boldsymbol{\Pi} \cdot \boldsymbol{\sigma},
$$

where $v_{\mathrm{F}}$ is the Fermi velocity, $\sigma=\left(\sigma_{x}, \sigma_{y}, \sigma_{z}\right)$ is the vector of Pauli matrices, and $\boldsymbol{\Pi}=\mathbf{k}+e \mathbf{A} / \hbar$ is the wave vector modulated by the vector potential $\mathbf{A}$. It is assumed that the Weyl nodes with chiralities $\chi= \pm$ are separated in the $z$ direction. For the magnetic field $\mathbf{B}=B(\sin \theta, 0, \cos \theta)$ applied in the $x-z$ plane, by the relation $\mathbf{B}=\boldsymbol{\nabla} \times \mathbf{A}$, we take the Landau gauge $\mathbf{A}=-B y\left(\cos \theta \hat{e}_{x}-\sin \theta \hat{e}_{z}\right)$. In this case, $k_{y}$ is no longer a

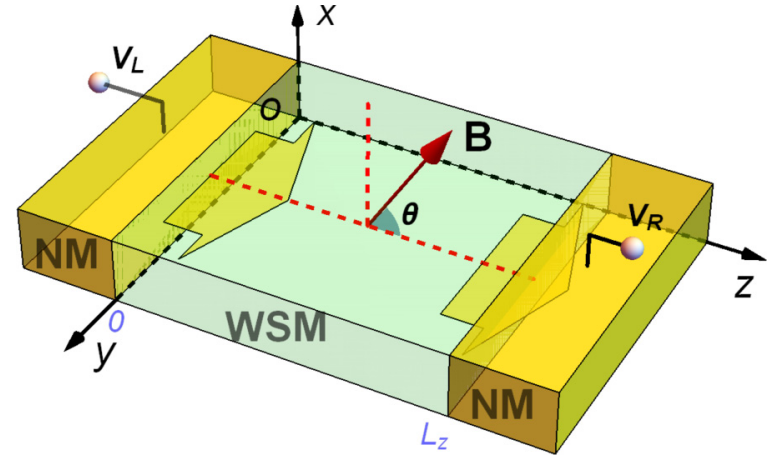

FIG. 1. Schematics of the setup, where two normal metal (NM) electrodes (yellow) with voltages $V_{L}$ and $V_{R}$ are attached to a $x$ - $z$ plane magnetic-field-driven WSM (cyan) at $z=0$ and $z=L_{z}$.

good quantum number, and should be treated as a quantum operator, i.e., $k_{y}=-i \partial_{y}$. By projecting the Hamiltonian into the Hilbert space expanded by the harmonic oscillator wave functions, one can solve for the eigenenergies of the LLs

$$
\varepsilon_{n}^{\chi}\left(k_{r}\right)=s_{n} \hbar \omega_{c} \sqrt{2|n|+\ell_{B}^{2} k_{r}^{2}}-\chi \hbar v_{\mathrm{F}} k_{r} \delta_{n, 0}
$$

with $k_{r}=k_{z} \cos \theta+k_{x} \sin \theta$, where $s_{n} \equiv \operatorname{sgn}(n)=\{-1,0,1\}$ for $n\{<,=,>\} 0, \omega_{c}=v_{\mathrm{F}} / \ell_{B}$, and $\ell_{B}=\sqrt{\hbar / e B}$ is the magnetic length. The energy spectrum of the LLs is plotted in Figs. 2(a) and 2(b). We see that the $n=0$ LLs are chiral, and all the $n \neq 0$ LLs are achiral. The slope of the LLs (a)

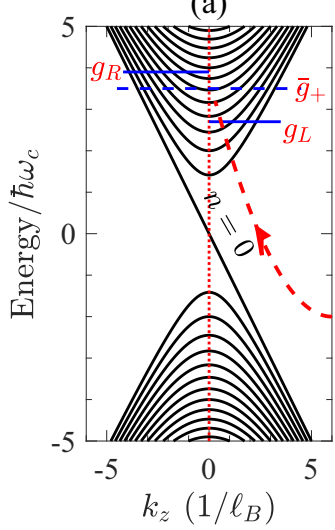

(b)

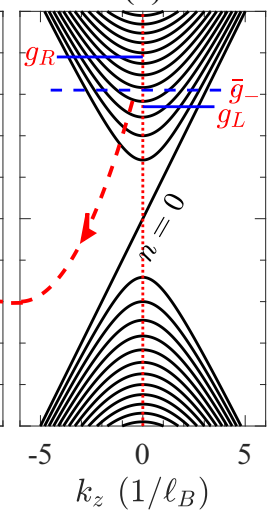

(c)

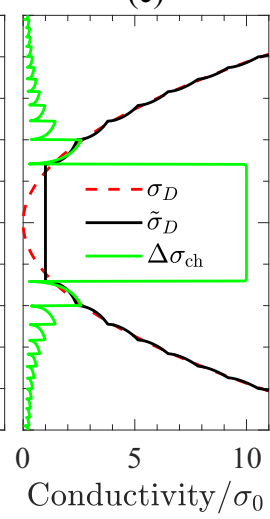

FIG. 2. The LLs in (a) $\chi=+$ and (b) $\chi=-$ Weyl valleys. (c) The calculated conductivities (horizontal axis) as functions of normalized energy $E_{\mathrm{F}} / \hbar \omega_{c}$ (vertical axis). The blue solid lines in (a) and (b) are an enlarged illustration of the fact that the forward (backward) moving Weyl fermions at the boundary with $v_{n}^{\chi}\left(k_{r}\right)>$ $(<) 0$ can only feel the chemical potential $g_{L(R)}=-e V_{L(R)}$ in the left (right) lead, which corresponds to the boundary conditions in Eqs. (11) and (12). The blue-dash lines sketch the different local equilibrium chemical potentials in the two Weyl valleys, originating from the intervalley fermion pumping effect due to the chiral anomaly, as indicated by the arrowed red-dashed curve. For convenience, in (c), we set $B=1 \mathrm{~T}, \tau_{\text {inter }} / \tau_{\text {intra }}=10$ and define $\sigma_{0}=$ $\left(2 e^{2} / h\right)\left\{e\left[(B=1 \mathrm{~T}) v_{\mathrm{F}} \tau_{\text {intra }}\right] / h\right\}$ as the unit of conductivity. 
corresponds to the electron group velocity

$$
v_{n}^{\chi}\left(k_{r}\right)=\frac{\partial \varepsilon_{n}^{\chi}\left(k_{r}\right)}{\hbar \partial k_{r}}= \begin{cases}-\chi v_{\mathrm{F}}, & n=0 \\ \hbar v_{\mathrm{F}}^{2} k_{r} / \varepsilon_{n}^{\chi}\left(k_{r}\right), & n \neq 0\end{cases}
$$

along the magnetic field, and the $\alpha=x, z$ components of the group velocity for the $n$th $\mathrm{LL}$ are given by $v_{\alpha, n}^{\chi}\left(k_{r}\right)=$ $v_{n}^{\chi}\left(k_{r}\right) \partial k_{r} / \partial k_{\alpha}$.

Let us consider the steady-state Boltzmann equation for the WSM

$$
v_{z, n}^{\chi} \frac{\partial f_{n}^{\chi}}{\partial z}=-\frac{f_{n}^{\chi}-f_{\chi}}{\tau_{\text {intra }}}-\frac{f_{n}^{\chi}-f_{g}}{\tau_{\text {inter }}},
$$

where $f_{n}^{\chi}$ is the electron distribution function for the $n$th LL in the $\chi$ valley, $f_{\chi}$ and $f_{g}=\left(f_{\chi}+f_{-\chi}\right) / 2$ denote, respectively, the local and global equilibrium electron distribution functions. In Eq. (4), we introduce the intervalley scattering term to the Boltzman equation phenomenologically, which is an analogy of the discussions of the weak localization in Ref. [44] and weak antilocalization in Ref. [45]. This approximation is widely adopted to investigate the magnetotransports in WSMs [46,47]. There also have been many works devoted to study of the impurity scattering mechanisms in WSMs, such as in Ref. [46]. The Weyl fermions in the two valleys are relaxed toward the local equilibrium distribution $f_{\chi}$ separately by the intravalley scattering, and toward the global equilibrium $f_{g}$ by the intervalley scattering. The scattering terms on the right-hand side of Eq. (4) account for the fact that the system is relaxed, simultaneously, toward local and global equilibrium by the intravalley and intervalley scattering, which are characterized by the relaxation times $\tau_{\text {intra }}$ and $\tau_{\text {inter }}$, respectively. For a relative small bias voltage between the electrodes, we can expand $f_{n}^{\chi}\left(k_{r}, z\right)=f_{0}\left(\varepsilon_{n}^{\chi}\right)+$ $\left[-\partial_{\varepsilon_{n}^{\chi}} f_{0}\left(\varepsilon_{n}^{\chi}\right)\right] g_{n}^{\chi}\left(k_{r}, z\right)$, where $g_{n}^{\chi}\left(k_{r}, z\right)$ describes the deviation of $f_{n}^{\chi}\left(k_{r}, z\right)$ from the equilibrium electron distribution function $f_{0}\left(\varepsilon_{n}^{\chi}\right)=1 /\left[e^{\left(\varepsilon_{n}^{\chi}-E_{F}\right) / k_{B} T}+1\right]$. The intravalley scattering is usually much stronger than the intervalley scattering, i.e., $\tau_{\text {intra }} \ll \tau_{\text {inter }}$, because of the momentum separation of the two Weyl nodes in the reciprocal space, which is also a necessary condition for the observation of the effect of the chiral anomaly [16]. Large ratios between the intravalley and intervalley scattering rates could come from the long-range Coulomb disorder. As estimated in Refs. [16,24], the ratio of the scattering timescales can be characterized by $\tau_{\text {inter }} / \tau_{\text {intra }} \sim$ $\left(2 k_{0} / k_{\mathrm{sc}}\right)^{4}$, where $2 k_{0}$ denotes momentum distance between the Weyl nodes and $k_{\mathrm{sc}}$ represents the screening wave vector. In the low-energy regime, $k_{\mathrm{sc}} \simeq k_{\mathrm{F}} \ll 2 k_{0}$ with $k_{\mathrm{F}}$ being the Fermi wave vector, and so large values of $\tau_{\text {inter }} / \tau_{\text {intra }}$ are possible. For example, if $k_{0} \simeq 2 k_{\mathrm{F}}, \tau_{\text {inter }} / \tau_{\text {intra }} \simeq 256$. For real WSM Na $3 \mathrm{Bi}$, the mean-free path $l_{\mathrm{a}} \equiv v_{\mathrm{F}} \tau_{\text {intra }}$ is conservatively estimated as about $10 \mathrm{~nm}$, while the intervalley relaxation lengths $l_{\mathrm{e}} \equiv v_{\mathrm{F}} \tau_{\text {inter }}$ can reach the order of tens of microns. Under this condition, the local equilibrium will be established every time before the intervalley scattering happens. Accordingly, it will be reasonable to replace $f_{n}^{\chi}$ with $f_{\chi}$ in the last term of Eq. (4), and obtain

$$
v_{z, n}^{\chi} \frac{\partial g_{n}^{\chi}\left(k_{r}, z\right)}{\partial z}=-\frac{g_{n}^{\chi}\left(k_{r}, z\right)-\bar{g}_{\chi}(z)}{\tau_{\text {intra }}}-\chi \frac{\Delta \bar{g}(z)}{\tau_{\text {inter }}},
$$

where $\Delta \bar{g}(z) \equiv \frac{\bar{g}_{+}(z)-\bar{g}_{-}(z)}{2}$ and $\bar{g}_{\chi}(z) \equiv\left\langle g_{n}^{\chi}\left(k_{r}, z\right)\right\rangle_{\chi}$. By definition, $\bar{g}_{\chi}(z)$ is essentially the local equilibrium chemical potential in the $\chi$ valley, and can be calculated by the momentum average at the local Fermi level [30]

$$
\langle\ldots\rangle_{\chi} \equiv \frac{\frac{1}{2 \pi \ell_{B}^{2}} \sum_{n, k_{r}} \int d \epsilon\left[-\partial_{\epsilon} f_{0}(\epsilon)\right] A_{\chi}\left(\epsilon, n, k_{r}\right)(\ldots)}{\frac{1}{2 \pi \ell_{B}^{2}} \sum_{n, k_{r}} \int d \epsilon\left[-\partial_{\epsilon} f_{0}(\epsilon)\right] A_{\chi}\left(\epsilon, n, k_{r}\right)},
$$

where $A_{\chi}\left(\epsilon, n, k_{r}\right)$ is the spectrum function with the impurityinduced level broadening considered, given by

$$
A_{\chi}\left(\epsilon, n, k_{r}\right)=-\frac{1}{\pi} \operatorname{Im}\left[\frac{1}{\epsilon+i \frac{\hbar}{2 \tau}-\varepsilon_{n}^{\chi}\left(k_{r}\right)}\right],
$$

with $1 / \tau=1 / \tau_{\text {intra }}+1 / \tau_{\text {inter }}$.

From Eq. (3), it is easy to find the identity $v_{z, n}^{\chi}\left(k_{r}\right)=$ $-v_{z, n}^{-\chi}\left(-k_{r}\right)$. By this symmetry, within the linear approximation $[48,49]$, we can find that the solution for the electron distribution function satisfies the condition

$$
\frac{\partial \bar{g}_{+}(z)}{\partial z}=\frac{\partial \bar{g}_{-}(z)}{\partial z}=\partial_{z} \bar{g}(z)
$$

with $\bar{g}(z) \equiv \frac{\bar{g}_{+}(z)+\bar{g}_{-}(z)}{2}$, such that $\partial_{z}[\Delta \bar{g}(z)]=0$. Consequently, $\Delta \bar{g}(z)$ may be considered as a constant, which will be denoted as $\Delta \bar{g}(z)=\Delta \mu$, and then $\bar{g}_{\chi}(z)$ must take the form $\bar{g}_{\chi}(z)=\bar{g}(z)+\chi \Delta \mu$, where $\Delta \mu$ is essentially the chiral chemical potential due to the chiral anomaly, which only can be relaxed by the intervalley scattering. In Figs. 2(a) and 2(b), $\bar{g}_{+}(z) \neq \bar{g}_{-}(z)$ are illustrated by the blue dashed lines. Averaging both sides of Eq. (5) results in

$$
\Delta \mu=\frac{\left\langle v_{z, n}^{-}\right\rangle_{-}-\left\langle v_{z, n}^{+}\right\rangle_{+}}{2} \tau_{\text {inter }} \partial_{z} \bar{g}(z) .
$$

We first carry out analytical calculation and discuss some of the properties of the particular solution given by Eq. (9), by considering the case for zero temperature and $\Gamma \equiv \hbar /(2 \tau) \rightarrow$ 0 . The results for the more general cases of finite temperatures and finite $\tau$ will be obtained by numerical calculations. Without loss of generality, we set $\theta=0$, so that $v_{z, n}^{\chi}=v_{n}^{\chi}$. It is easy to derive $\left\langle v_{n}^{\chi}\right\rangle_{\chi}=h^{-1} \Delta N_{\mathrm{ch}}^{\chi} / v_{\chi}\left(E_{\mathrm{F}}\right)$, where $v_{\chi}\left(E_{\mathrm{F}}\right)=$ $\Theta / 2 \pi \ell_{B}^{2} h v_{\mathrm{F}} \equiv \nu\left(E_{\mathrm{F}}\right)$ is the density of states (DOS) in the $\chi$ valley at the Fermi level, with

$$
\Theta=2 \sum_{n=0}^{n_{c}} \frac{1}{\sqrt{1-2|n|\left(\hbar \omega_{c} / E_{\mathrm{F}}\right)^{2}}}-1 .
$$

Here, $n_{c}=\operatorname{sgn}\left(E_{\mathrm{F}}\right)\left\lfloor E_{\mathrm{F}}^{2} / 2\left(\hbar \omega_{c}\right)^{2}\right\rfloor$ labels the highest (lowest) LL crossed by the Fermi level for $E_{\mathrm{F}}>0\left(E_{\mathrm{F}}<0\right)$, with $\lfloor\cdot\rfloor$ representing the rounding down operation. Owing to the factor $\Theta$, the DOS oscillates strongly with varying $E_{\mathrm{F}}$ or $B$, exhibiting the van Hove singularities at $E_{\mathrm{F}}=$ $\operatorname{sgn}(n) \sqrt{2|n|} \hbar \omega_{c}$. This results in the periodic-in-1/B quantum oscillations of the chiral chemical potential [30]. Here, $\Delta N_{\mathrm{ch}}^{\chi}=\frac{1}{2 \pi \ell_{B}^{2}} \sum_{\left\{k_{r}=k_{r, n}^{\chi}(i)\right\}} \operatorname{sgn}\left(v_{n}^{\chi}\right)$, where the summation is to add up the signs of the group velocity $v_{n}^{\chi}$ at all the intersection points between the Fermi level and LLs in the $\chi$ valley. By definition, $\Delta N_{\mathrm{ch}}^{\chi}$ is essentially the number difference between the forward and backward moving channels at $E_{\mathrm{F}}$ in the $\chi$ valley per unit cross section. For the achiral $n \neq 0$ LLs, the forward and backward moving channels are 
always in pairs, making zero contribution to $\Delta N_{\mathrm{ch}}^{\chi}$. Therefore, $\Delta N_{\mathrm{ch}}^{\chi}$ equals to the number of chiral channels in the $n=$ 0 LL with its sign determined by the propagating direction of the chiral channels, namely, $\Delta N_{\mathrm{ch}}^{\chi}=-\chi / 2 \pi \ell_{B}^{2}$. Subsequently, we obtain $\left\langle v_{n}^{+}\right\rangle_{+}=-\left\langle v_{n}^{-}\right\rangle_{-}=-v_{\mathrm{F}} / \Theta$ and then $\Delta \mu=\left(v_{\mathrm{F}} \tau_{\text {inter }} / \Theta\right) \partial_{z} \bar{g}(z)$.

To proceed, we consider the fact that at the boundaries, the right- (left-) moving Weyl fermions with $v_{z, n}^{\chi}\left(k_{r}\right)>(<) 0$ can only feel the chemical potential $g_{L(R)}=-e V_{L(R)}$ in the left (right) electrode, which are illustrated by the blue solid lines in Figs. 2(a) and 2(b). As a result, we impose the following boundary conditions to the inhomogeneous differential (5), for the left interface $(z=0)$ and right interface $\left(z=L_{z}\right)$ between the sample and the electrodes:

$$
\begin{array}{ll}
g_{n}^{\chi}\left(k_{r}, z=0\right)=-e V_{L} \equiv g_{L} & \text { for } v_{z, n}^{\chi}>0, \\
g_{n}^{\chi}\left(k_{r}, z=L_{z}\right)=-e V_{R} \equiv g_{R} & \text { for } v_{z, n}^{\chi}<0 .
\end{array}
$$

Then, we can obtain for the general solution to differential (5)

$$
\begin{aligned}
g_{n}^{\chi}\left(k_{r}, z\right)= & \theta\left(v_{z, n}^{\chi}\right) g_{L} e^{-\lambda(0)}+\theta\left(-v_{z, n}^{\chi}\right) g_{R} e^{-\lambda\left(L_{z}\right)} \\
& +\int_{0}^{z} \theta\left(v_{z, n}^{\chi}\right)\left[\frac{\bar{g}_{\chi}(\xi)-\chi \frac{\tau_{\text {intra }}}{\tau_{\text {inter }}} \Delta \bar{g}(\xi)}{v_{z, n}^{\chi} \tau_{\text {intra }}}\right] e^{-\lambda(\xi)} d \xi \\
& +\int_{L_{z}}^{z} \theta\left(-v_{z, n}^{\chi}\right)\left[\frac{\bar{g}_{\chi}(\xi)-\chi \frac{\tau_{\text {intra }}}{\tau_{\text {inter }}} \Delta \bar{g}(\xi)}{v_{z, n}^{\chi} \tau_{\text {intra }}}\right] e^{-\lambda(\xi)} d \xi,
\end{aligned}
$$

where $\lambda(\xi) \equiv(z-\xi) / v_{z, n}^{\chi} \tau_{\text {intra }}$ and $\theta(x)$ is the Heaviside function. We will make the linear approximation for $\bar{g}_{\chi}(\xi)$, which has been shown to be very close to the exact numerical solution [48,49]. Replacing $\bar{g}_{\chi}(\xi)=a_{\chi}+b \xi$ and $\Delta \bar{g}(\xi)=$ $\Delta \mu$ in Eq. (13) and performing the integral, we arrive at

$$
\begin{aligned}
g_{n}^{\chi}\left(k_{r}, z\right)= & \bar{g}_{\chi}(z)-w_{\chi}+\theta\left(v_{z, n}^{\chi}\right) e^{-\lambda(0)}\left(g_{L}-a_{\chi}+w_{\chi}\right) \\
& +\theta\left(-v_{z, n}^{\chi}\right) e^{-\lambda\left(L_{z}\right)}\left(g_{R}-a_{\chi}-b L_{z}+w_{\chi}\right)
\end{aligned}
$$

with $a_{\chi}=a+\chi \Delta \mu$ and $w_{\chi}=\left(b v_{z, n}^{\chi}+\chi \Delta \mu / \tau_{\text {inter }}\right) \tau_{\text {intra }}$. By averaging both sides of Eq. (14), we can obtain the selfconsistent equations for determining $a$ and $b$. It should be noted that the linear approximation is most accurate around the middle of the system [48,49]. Once the electron distribution function $g_{n}^{\chi}\left(k_{r}, z\right)$ is determined, the electrical current density, in principle, can be calculated at any cross section. Here, it is convenient to use the linear approximation, and calculate the electrical current density at the $z=L_{z} / 2$ cross section $[48,49]$

$$
j_{\alpha}=\frac{-e}{2 \pi \ell_{B}^{2}} \sum_{\chi, n} \int_{-\infty}^{\infty} v_{\alpha, n}^{\chi}\left(k_{r}\right) g_{n}^{\chi}\left(k_{r}, \frac{L_{z}}{2}\right)\left[-\frac{\partial f_{0}\left(\varepsilon_{n}^{\chi}\right)}{\partial \varepsilon_{n}^{\chi}}\right] \frac{d k_{r}}{2 \pi} .
$$

\section{RESULTS AND DISCUSSIONS}

If we rotate the $z$ axis to align with the magnetic field, the resistivity tensor can be expressed as [50]

$$
\left(\begin{array}{c}
E_{\perp} \\
E_{\|}
\end{array}\right)=\left(\begin{array}{cc}
\rho_{\perp} & 0 \\
0 & \rho_{\|}
\end{array}\right)\left(\begin{array}{c}
j_{\perp} \\
j_{\|}
\end{array}\right)
$$

with $\rho_{\|(\perp)}=1 / \sigma_{\|(\perp)}$ corresponding to the current flow parallel (vertical) to the direction of the magnetic field. By the transforms $\left(j_{\perp}, j_{\|}\right)^{T}=\hat{R}\left(j_{x}, j_{z}\right)^{T}$ and $\left(E_{\perp}, E_{\|}\right)^{T}=$ $\hat{R}\left(E_{x}, E_{z}\right)^{T}$, where

$$
\hat{R}=\left(\begin{array}{cc}
\cos \theta & -\sin \theta \\
\sin \theta & \cos \theta
\end{array}\right)
$$

is the rotation matrix about the $y$ axis, it is easy to obtain the general relation $\left(E_{x}, E_{z}\right)^{T}=\rho\left(j_{x}, j_{z}\right)^{T}$, in which

$$
\rho=\left(\begin{array}{cc}
\rho_{\|}+\Delta \rho \cos ^{2} \theta & -\Delta \rho \sin \theta \cos \theta \\
-\Delta \rho \sin \theta \cos \theta & \rho_{\perp}-\Delta \rho \cos ^{2} \theta
\end{array}\right)
$$

is the resistivity tensor in the original coordinate system and $\Delta \rho=\rho_{\perp}-\rho_{\|}$is the magnetic-field-induced resistivity anisotropy. For most metals, as the magnetic field increases, the vertical conductivity $\sigma_{\perp} \sim \sigma_{D} /\left(1+\mu^{2} B^{2}\right)$ will decrease gradually, where $\mu$ denotes the mobility $[33,37]$ and $\sigma_{D}=$ $n_{e} e^{2} v_{\mathrm{F}} \tau_{\text {intra }} /\left(\hbar k_{\mathrm{F}}\right)$ is the zero-field Drude conductivity [30] with $k_{\mathrm{F}}$ and $n_{e}$, being, respectively, the Fermi wave vector and carrier density. The parallel conductivity $\sigma_{\|}$, usually, responses differently from $\sigma_{\perp}$ to the magnetic field. In this sense, the planar Hall conductivity can be easily understood. It can be expected that, for a normal metal, with increasing the magnetic field, the amplitude of the planar Hall conductivity $\Delta \sigma=\sigma_{\|}-\sigma_{\perp}$ would first increase and finally tend to a saturation value. However, as we will show, in WSMs, due to the chiral anomaly, the amplitude of the planar Hall conductivity will be unsaturated and sharply peak around $\theta=0$.

The parallel conductivity $\sigma_{\|}$, which includes contribution of the chiral anomaly, can be obtained through $j_{z}$ by setting $\theta=0$. In the following, we would discuss some representative limiting cases.

(i) The ballistic limit, i.e., $L_{z} \ll v_{\mathrm{F}} \tau_{\text {intra }}$. It is easy to see that the first and second terms in Eq. (13) are of the zeroth order of $L_{z} / v_{\mathrm{F}} \tau_{\text {intra }}$, and the third and fourth terms are of the linear order of $L_{z} / v_{\mathrm{F}} \tau_{\text {intra }}$ and so can be omitted. Therefore, the electron distribution function reduces to $g_{n}^{\chi}\left(k_{r}, z\right)=\theta\left(v_{n}^{\chi}\right) g_{L}+$ $\theta\left(-v_{n}^{\chi}\right) g_{R}$, which takes a simple transmission form. The parallel conductivity is derived to be

$$
\sigma_{\|} \equiv \frac{j_{z} L_{z}}{V_{L}-V_{R}}=\frac{e^{2}}{h} N_{\mathrm{ch}} L_{z}
$$

with $N_{\text {ch }} \equiv \frac{1}{2 \pi \ell_{B}^{2}} \sum_{\chi, n} \theta\left(v_{n}^{\chi}\right)=\frac{1}{2 \pi \ell_{B}^{2}} \sum_{\chi, n} \theta\left(-v_{n}^{\chi}\right)$, where the summation runs over all the intersection points, denoted by $\left\{k_{r}=k_{r, n}^{\chi}(i)\right\}$, between the Fermi level $E_{\mathrm{F}}$ and LLs in the two valleys. Equation (19) is just the well-known Landauer-Büttiker formula and $N_{\text {ch }}$ is the total number of forward-moving $\left(v_{n}^{\chi}>0\right)$ or backward-moving $\left(v_{n}^{\chi}<0\right)$ open channels per unit cross section at the Fermi level. The total numbers of open channels moving in the two directions are apparently equal. In the ballistic limit, the electrical conductivity displays stepwise behavior with changing the Fermi energy due to the Landau quantization, as in an ordinary electron system, without showing the chiral anomaly.

(ii) The diffusive limit, i.e., $L_{z} \gg v_{\mathrm{F}} \tau_{\text {intra }}$. Setting $\chi=+$ and - , Eq. (14) gives two coupled equations. Adding up the two equations and setting $z=L_{z} / 2$, we derive

$$
0=\left(g_{L}+g_{R}\right)-2 a-b L_{z} .
$$


The first-order derivative of Eq. (14) with respect to $z$ at $z=$ $L_{z} / 2$ gives another equation

$$
0=\left(g_{L}-g_{R}\right)+b L_{z}+2 \Delta \mu .
$$

Combining Eqs. (9) and (20) and (21), we can arrive at

$$
\Delta \mu=e E_{\|} l_{\mathrm{e}} \frac{1}{\Theta+2 l_{\mathrm{e}} / L_{z}},
$$

where $E_{\|} \equiv\left(V_{L}-V_{R}\right) / L_{z}$. The parameter $a$ is a gaugedependent parameter and will not affect the conductivity since a different choice of the zero point of the electric potential only shifts both $\bar{g}_{+}(z)$ and $\bar{g}_{-}(z)$ by a same constant. To the leading order in $\tau_{\text {intra }} / \tau_{\text {inter }} \ll 1$, we can derive $\sigma_{\|}=\sigma_{D}+$ $\Delta \sigma_{D}+\Delta \sigma_{\mathrm{ch}}$, where

$$
\Delta \sigma_{\mathrm{ch}}=\frac{e}{h}\left(\Delta N_{\mathrm{ch}}^{-}-\Delta N_{\mathrm{ch}}^{+}\right) \frac{\Delta \mu}{E_{\|}}
$$

and $\Delta \sigma_{D}=\tilde{\sigma}_{D}-\sigma_{D}$, with $\tilde{\sigma}_{D}$ being the magnetic field renormalized Drude conductivity. This formula illuminates the fact that the anomalous LMC comes from the chiral chemical potential $\Delta \mu$ driving extra electrical current to flow through the totally $\left(\Delta N_{\mathrm{ch}}^{-}-\Delta N_{\mathrm{ch}}^{+}\right.$) chiral channels in the $n=0$ LLs. Equation (23) can be further derived into

$$
\Delta \sigma_{\mathrm{ch}}=\frac{2 e^{2}}{h} \frac{e B}{h} \frac{v_{\mathrm{F}} \tau_{\text {inter }}}{\Theta+2 l_{\mathrm{e}} / L_{z}}
$$

and the renormalized Drude conductivity reads as

$$
\tilde{\sigma}_{D}=\frac{2 e^{2}}{h} \frac{e B}{h} \frac{\vartheta}{1+2 \Theta^{-1} l_{\mathrm{e}} / L_{z}} v_{\mathrm{F}} \tau_{\mathrm{intra}},
$$

where, for low temperatures,

$$
\vartheta=2 \sum_{n=0}^{n_{c}} \sqrt{1-2|n|\left(\hbar \omega_{c} / E_{\mathrm{F}}\right)^{2}}-1 .
$$

According to Eq. (18), we obtain for the LMC $[40,41]$

$$
\Delta \sigma_{z z} \equiv \rho_{z z}^{-1}(B)-\rho_{z z}^{-1}(0)=\frac{\Delta \sigma \cos ^{2} \theta}{1+\left(\Delta \sigma / \sigma_{\perp}\right) \sin ^{2} \theta},
$$

where, for low energies, $\quad\left|\sigma_{\perp}-\sigma_{D}\right|<\sigma_{D}=\frac{2 e^{2}}{h}$ $\left[E_{\mathrm{F}} /\left(\sqrt{3 \pi} \hbar v_{\mathrm{F}}\right)\right]^{2} v_{\mathrm{F}} \tau_{\text {intra }}$ was neglected. As can be seen, if $\Delta \sigma \ll \sigma_{\perp}$, the LMC $\Delta \sigma_{z z} \simeq \Delta \sigma \cos ^{2} \theta$ follows the regular angular dependence, whose amplitude increases with the increment on $B$, while if the prefactor of $\sin ^{2} \theta$ is large, as $\theta$ increases from $0, \Delta \sigma_{z z}$ will, gradually, deviate from the $\cos ^{2} \theta$ dependence and tend to the $\cot ^{2} \theta$ dependence, and then leads to an angular narrowing behavior of the LMC around $\theta=0$.

In the chiral diffusive limit $l_{\mathrm{e}} \ll L_{z}$, Eq. (24) reproduces the quantum formula given by Ref. [30], which predicts the $1 / B$-quantum oscillations for the positive LMC. In the chiral ballistic limit, i.e., $l_{\mathrm{e}} / L_{z} \gg \Theta / 2$, the chiral chemical potential is determined by the actual relaxation length of the chirality, i.e., the length of the sample, which is in accord with our intuition. As seen from Fig. 2(c), both $\tilde{\sigma}_{D}$ and $\Delta \sigma_{\text {ch }}$ display a plateau for $\left|E_{\mathrm{F}}\right|<\sqrt{2} \hbar \omega_{c}$, which is contributed only from the chiral $n=0$ LL. If $\left|E_{\mathrm{F}}\right|<\sqrt{3 / 2} \hbar \omega_{c}, \tilde{\sigma}_{D}>\sigma_{D}$ always, which contributes a normal $B$-linearly term $\Delta \sigma_{D}$ to the positive LMC. This implies that the chiral $n=0 \mathrm{LL}$ can contribute to the positive LMC through two different ways: one is directly by increasing the topological transport channels since the $n=0$ LLs with degeneracy $B$-linearly dependent are always crossed by the Fermi level, and the other is indirectly by the chiral anomaly. The former will be greatly weakened by the achiral $n \neq 0$ LLs, as shown by the dark solid curve in Fig. 2(c), where, because of $E_{\mathrm{F}}$-dependent group velocities of the $n \neq 0$ LLs, $\tilde{\sigma}_{D}$ displays a wavy stepwise behavior along $\sigma_{D}$ with increasing the Fermi level. In contrast, $\Delta \sigma_{\mathrm{ch}}$ exhibits drastic quantum oscillations with respect to $E_{\mathrm{F}}$, whose amplitude decays with a rate $\propto 1 / E_{\mathrm{F}}^{2}$. In WSMs, due to separation of the Weyl nodes, $l_{\mathrm{e}} \gg l_{\mathrm{a}}$ and so $\Delta \sigma_{\mathrm{ch}} \gg \Delta \sigma_{D}$. As a consequence, $\Delta \sigma_{\mathrm{ch}}$ will dominate the positive LMC.

For $\left|E_{\mathrm{F}}\right| \gg \hbar \omega_{c}$, being equivalent to the low magnetic field limit, $\Theta \simeq 2\left(E_{\mathrm{F}} / \hbar \omega_{c}\right)^{2}$ and $\tilde{\sigma}_{D} \simeq \sigma_{D}$. In this classical limit, $\Delta \sigma_{z z}$ can be reduced to be

$$
\Delta \sigma_{z z}=\frac{e^{2}}{4 \pi^{2} \hbar} \frac{(e B)^{2} v_{\mathrm{F}}^{2}}{E_{\mathrm{F}}^{2}} v_{\mathrm{F}} \tau_{\text {inter }} \frac{\cos ^{2} \theta}{1+\frac{3}{4}\left(l_{\mathrm{e}} / l_{\mathrm{a}}\right)\left(\hbar \omega_{c} / E_{\mathrm{F}}\right)^{4} \sin ^{2} \theta} .
$$

As can be seen, if $\hbar \omega_{c} \ll E_{\mathrm{F}}\left(\frac{4 l_{\mathrm{a}}}{3 l_{\mathrm{e}}}\right)^{1 / 4}, \Delta \sigma_{z z} \propto \cos ^{2} \theta$ returns to the regular angular dependence and, moreover, for $\theta=$ $0, \Delta \sigma_{z z}$ recovers the classical positive LMC formula in Refs. [27,29,30]. For $\left|E_{\mathrm{F}}\right|<\sqrt{2} \hbar \omega_{c}$, equivalent to the strong magnetic field limit, $\Theta=1$ and

$$
\Delta \sigma_{z z}=\frac{2 e^{2}}{h} \frac{e B}{h} v_{\mathrm{F}} \tau_{\text {inter }} \frac{\cos ^{2} \theta}{1+\frac{3}{2}\left(l_{\mathrm{e}} / l_{\mathrm{a}}\right)\left(\hbar \omega_{c} / E_{\mathrm{F}}\right)^{2} \sin ^{2} \theta}
$$

crosses over to the ultraquantum limit. In the limiting case $\hbar \omega_{c} \gg E_{\mathrm{F}} \sqrt{l_{\mathrm{a}} / l_{\mathrm{e}}}, \Delta \sigma_{z z}$ will exhibit the angular narrowing (a)

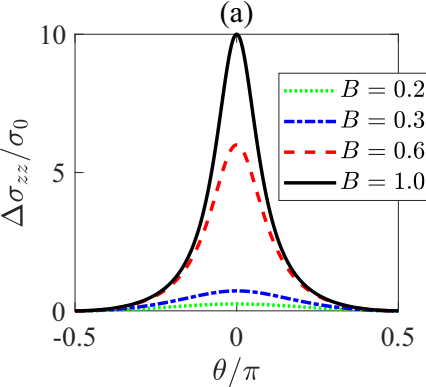

(c)

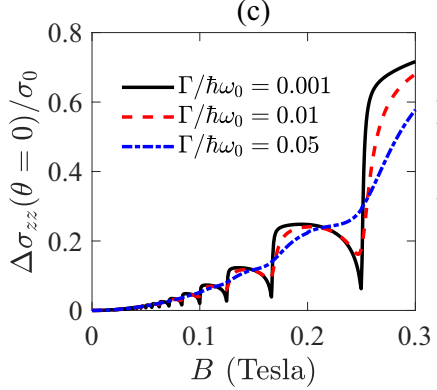

(b)

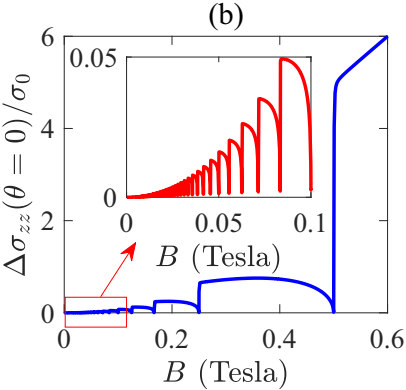

(d)

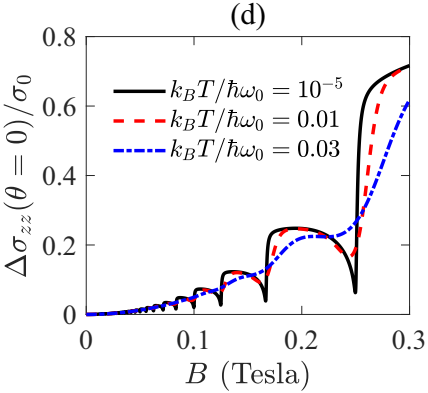

FIG. 3. (a) $\Delta \sigma_{z z}$ as a function of $\theta$ for varied $B$. (b) - (d) $\Delta \sigma_{z z}(\theta=$ 0 ) as a function of $B$, respectively, for $\Gamma=k_{B} T=10^{-5} \hbar \omega_{0}$, varied $\Gamma$ with $k_{B} T=10^{-5} \hbar \omega_{0}$, and varied $k_{B} T$ with $\Gamma=10^{-3} \hbar \omega_{0}$. The inset of (b) is the data in the low magnetic region replotted to show the quantum oscillations of the LMC. Here, for convenience, we set $\hbar \omega_{0}=v_{\mathrm{F}} \sqrt{e \hbar[B=1 \mathrm{~T}]}$ as the unit of energy. Other parameters are the same as Fig. 2(c). The results for finite temperatures and finite level broadening are obtained from numerical calculations. 


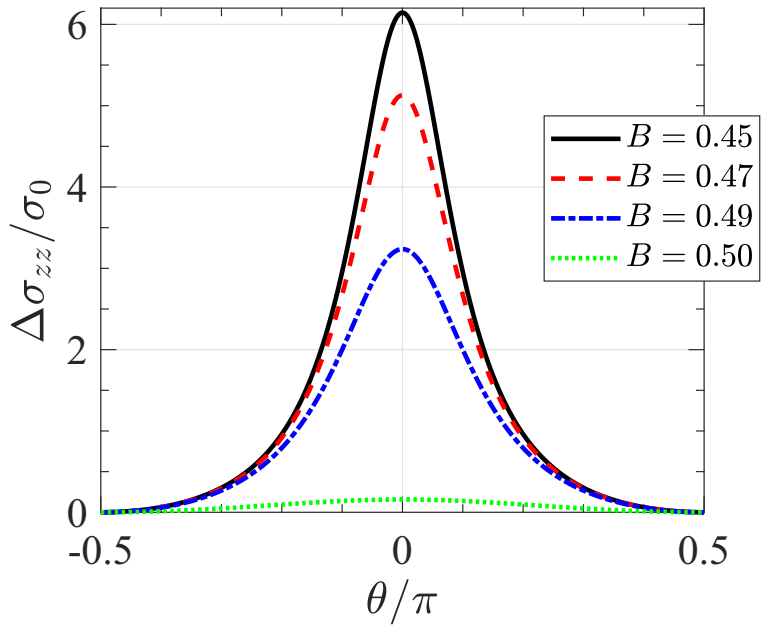

FIG. 4. Magnetic field dependence of the angular narrowing behavior in contrast to that in Fig. 3(a). Here, we chose $l_{\mathrm{e}} / l_{\mathrm{a}}=100$ and other parameters are the same as Fig. 3.

phenomenon, with the amplitude tunable by the magnetic field. Also, for $\theta=0$, the LMC returns to the quantum formula derived in Ref. [30]. The above analyses are confirmed by the numerical results plotted in Figs. 3(a) and 4.

As shown by Figs. 3(b)-3(d), the quantum oscillations of the LMC are quite sensitive to the impurity scattering scaled by $\Gamma=\hbar /(2 \tau)$ and the temperature, due to broadening of the LLs and smearing of the Fermi level. The quantum oscillations are resolvable only when the spacing of the LLs is much greater than the impurity-induced broadening [24] $\Gamma$ or thermal fluctuation energy $k_{B} T$. Therefore, the LMC may exhibit other anomalous behaviors, especially for a relatively weak magnetic field. For instance, for a fixed magnetic field strength, the LMC can increase or decrease with increasing temperature or strength of the impurity scattering, depending on whether the field strength is near a valley or near a peak, while, for a fixed Fermi energy, if increasing the magnetic field pushes a valley to the Fermi level, the height of the LMC would decrease, drastically, as shown by Fig. 4. This could explain the anomalous magnetic field dependence of the angular narrowing phenomenon observed in Ref. [37]. For relatively strong magnetic fields, the effects of the impurity scattering and thermal fluctuations would be less important.
As discussed in Ref. [28], because the momentum space in the condensed-matter context being confined to the first Brillouin zone is compact, a real microscopic model of WSM does not actually possess exact chiral symmetry. However, the $n=0$ LLs in WSMs, protected by the topological nature of the system, always come in pairs with opposite group velocity directions corresponding to monopole charges of the Weyl nodes, which breaks the symmetry of the number of forwardand backward-moving channels. In equilibrium, the positive LMC is expected to be vanishing since no chemical potential difference can exist among the Weyl valleys. However, upon application of an additional electric field, a nonzero chemical potential difference, due to violation of the channel symmetry, can be established among the Weyl valleys by nonequilibrium processes, which leads to the emergence of the positive LMC. While the chiral anomaly in condensed-matter context might be less well defined than that in the context of relativistic field theory, the positive LMC in WSMs could be understood as a consequence of violation of the channel symmetry.

\section{SUMMARY}

We studied the chiral anomaly in a finite-size WSM for both the ballistic and diffusive limits, and derived an analytical formula for the chiral-anomaly-induced positive LMC. It is found that the positive LMC comes from the chiral channels in the $n=0$ LLs and will exhibit the angular narrowing if $\hbar \omega_{c} \gg E_{\mathrm{F}} \sqrt{l_{\mathrm{a}} / l_{\mathrm{e}}}$. This suggests that the behavior of the angular narrowing, in addition to the relaxation lengths, can be modulated by the relative magnitudes of the Fermi energy and magnetic field. The anomalous magnetic field dependence of the angular narrowing can be associated with quantum oscillations of the chiral anomaly. Our findings are helpful to understand the angular narrowing behaviors of the positive LMC in WSMs.

\section{ACKNOWLEDGMENTS}

This work was supported by the National Natural Science Foundation of China under Grants No. 11904107 (M.X.D.), No. 11674160 (L.S.), No. 11874016 (R.Q.W.), No. 11574155 (R.M.), and No. 11804130 (W.L.), by the Guangdong NSF of China under Grant No. 2020A1515011566 (M.X.D) and the Key Program for Guangdong NSF of China under Grants No. 2017B030311003 and No. GDUPS(2017) and the projects funded by South China Normal University.
[1] X. Wan, A. M. Turner, A. Vishwanath, and S. Y. Savrasov, Phys. Rev. B 83, 205101 (2011).

[2] K.-Y. Yang, Y.-M. Lu, and Y. Ran, Phys. Rev. B 84, 075129 (2011).

[3] G. Xu, H. Weng, Z. Wang, X. Dai, and Z. Fang, Phys. Rev. Lett. 107, 186806 (2011).

[4] S. M. Young, S. Zaheer, J. C. Y. Teo, C. L. Kane, E. J. Mele, and A. M. Rappe, Phys. Rev. Lett. 108, 140405 (2012).

[5] Z. Wang, H. Weng, Q. Wu, X. Dai, and Z. Fang, Phys. Rev. B 88, 125427 (2013).
[6] Z. K. Liu, B. Zhou, Y. Zhang, Z. J. Wang, H. M. Weng, D. Prabhakaran, S.-K. Mo, Z. X. Shen, Z. Fang, X. Dai, Z. Hussain, and Y. L. Chen, Science 343, 864 (2014).

[7] M. Neupane, S.-Y. Xu, R. Sankar, N. Alidoust, G. Bian, C. Liu, I. Belopolski, T.-R. Chang, H.-T. Jeng, H. Lin, A. Bansil, F. Chou, and M. Z. Hasan, Nat. Commun. 5, 3786 (2014).

[8] S.-Y. Xu, I. Belopolski, N. Alidoust, M. Neupane, G. Bian, C. Zhang, R. Sankar, G. Chang, Z. Yuan, C.-C. Lee, S.-M. Huang, H. Zheng, J. Ma, D. S. Sanchez, B. Wang, A. Bansil, F. Chou, P. P. Shibayev, H. Lin, S. Jia, and M. Z. Hasan, Science 349, 613 (2015). 
[9] B. Q. Lv, N. Xu, H. M. Weng, J. Z. Ma, P. Richard, X. C. Huang, L. X. Zhao, G. F. Chen, C. E. Matt, F. Bisti, V. N. Strocov, J. Mesot, Z. Fang, X. Dai, T. Qian, M. Shi, and H. Ding, Nat. Phys. 11, 724 (2015).

[10] B. Q. Lv, H. M. Weng, B. B. Fu, X. P. Wang, H. Miao, J. Ma, P. Richard, X. C. Huang, L. X. Zhao, G. F. Chen, Z. Fang, X. Dai, T. Qian, and H. Ding, Phys. Rev. X 5, 031013 (2015).

[11] L. Lu, Z. Wang, D. Ye, L. Ran, L. Fu, J. D. Joannopoulos, and M. Soljačić, Science 349, 622 (2015).

[12] G. E. Volovik, The Universe in a Helium Droplet, Vol. 117 (Oxford University Press, Oxford, 2003).

[13] H. Nielsen and M. Ninomiya, Phys. Lett. B 130, 389 (1983).

[14] M. N. Ali, J. Xiong, S. Flynn, J. Tao, Q. D. Gibson, L. M. Schoop, T. Liang, N. Haldolaarachchige, M. Hirschberger, N. Ong, and R. Cava, Nature (London) 514, 205 (2014).

[15] C. Shekhar, A. K. Nayak, Y. Sun, M. Schmidt, M. Nicklas, I. Leermakers, U. Zeitler, Y. Skourski, J. Wosnitza, Z. Liu, Y. Chen, W. Schnelle, H. Borrmann, Y. Grin, C. Felser, and B. Yan, Nat. Phys. 11, 645 (2015).

[16] S. A. Parameswaran, T. Grover, D. A. Abanin, D. A. Pesin, and A. Vishwanath, Phys. Rev. X 4, 031035 (2014).

[17] N. P. Armitage, E. J. Mele, and A. Vishwanath, Rev. Mod. Phys. 90, 015001 (2018).

[18] D.-W. Zhang, S.-L. Zhu, and Z. D. Wang, Phys. Rev. A 92, 013632 (2015).

[19] D.-W. Zhang, Y.-Q. Zhu, Y. X. Zhao, H. Yan, and S.-L. Zhu, Adv. Phys. 67, 253 (2018).

[20] X.-S. Li, C. Wang, M.-X. Deng, H.-J. Duan, P.-H. Fu, R.-Q. Wang, L. Sheng, and D. Y. Xing, Phys. Rev. Lett. 123, 206601 (2019).

[21] M.-X. Deng, Y.-Y. Yang, W. Luo, R. Ma, C.-Y. Zhu, R.-Q. Wang, L. Sheng, and D. Y. Xing, Phys. Rev. B 100, 235105 (2019).

[22] M. Yang, Q.-T. Hou, and R.-Q. Wang, New J. Phys. 21, 113057 (2019).

[23] M. Yang, Q.-T. Hou, and R.-Q. Wang, New J. Phys. 22, 033015 (2020).

[24] Y.-Y. Yang, M.-X. Deng, H.-J. Duan, W. Luo, and R.-Q. Wang, Phys. Rev. B 101, 205137 (2020).

[25] L. X. Yang, Z. K. Liu, Y. Sun, H. Peng, H. F. Yang, T. Zhang, B. Zhou, Y. Zhang, Y. F. Guo, M. Rahn, D. Prabhakaran, Z. Hussain, S. K. Mo, C. Felser, B. Yan, and Y. L. Chen, Nat. Phys. 11, 728 (2015).

[26] M.-X. Deng, W. Luo, R.-Q. Wang, L. Sheng, and D. Y. Xing, Phys. Rev. B 96, 155141 (2017).

[27] D. T. Son and B. Z. Spivak, Phys. Rev. B 88, 104412 (2013).

[28] A. A. Burkov, Phys. Rev. Lett. 113, 247203 (2014).

[29] A. A. Burkov, Phys. Rev. B 91, 245157 (2015).
[30] M.-X. Deng, G. Y. Qi, R. Ma, R. Shen, R.-Q. Wang, L. Sheng, and D. Y. Xing, Phys. Rev. Lett. 122, 036601 (2019).

[31] J. Xiong, S. K. Kushwaha, T. Liang, J. W. Krizan, M. Hirschberger, W. Wang, R. J. Cava, and N. P. Ong, Science 350, 413 (2015).

[32] C.-Z. Li, L.-X. Wang, H. Liu, J. Wang, Z.-M. Liao, and D.-P. Yu, Nat. Commun. 6, 10137 (2015).

[33] Y. Wang, E. Liu, H. Liu, Y. Pan, L. Zhang, J. Zeng, Y. Fu, M. Wang, K. Xu, Z. Huang, Z. Wang, H.-Z. Lu, D. Xing, B. Wang, X. Wan, and F. Miao, Nat. Commun. 7, 13142 (2016).

[34] Y.-Y. Lv, X. Li, B.-B. Zhang, W. Y. Deng, S.-H. Yao, Y. B. Chen, J. Zhou, S.-T. Zhang, M.-H. Lu, L. Zhang, M. Tian, L. Sheng, and Y.-F. Chen, Phys. Rev. Lett. 118, 096603 (2017).

[35] X. Huang, L. Zhao, Y. Long, P. Wang, D. Chen, Z. Yang, H. Liang, M. Xue, H. Weng, Z. Fang, X. Dai, and G. Chen, Phys. Rev. X 5, 031023 (2015).

[36] H. Li, H. He, H.-Z. Lu, H. Zhang, H. Liu, R. Ma, Z. Fan, S.-Q. Shen, and J. Wang, Nat. Commun. 7, 10301 (2016).

[37] C.-L. Zhang, S.-Y. Xu, I. Belopolski, Z. Yuan, Z. Lin, B. Tong, G. Bian, N. Alidoust, C.-C. Lee, S.-M. Huang, T.-R. Chang, G. Chang, C.-H. Hsu, H.-T. Jeng, M. Neupane, D. S. Sanchez, H. Zheng, J. Wang, H. Lin, C. Zhang et al., Nat. Commun. 7, 10735 (2016).

[38] Y. Zhao, H. Liu, J. Yan, W. An, J. Liu, X. Zhang, H. Wang, Y. Liu, H. Jiang, Q. Li, Y. Wang, X.-Z. Li, D. Mandrus, X. C. Xie, M. Pan, and J. Wang, Phys. Rev. B 92, 041104(R) (2015).

[39] S. Nandy, G. Sharma, A. Taraphder, and S. Tewari, Phys. Rev. Lett. 119, 176804 (2017).

[40] A. A. Burkov and Y. B. Kim, Phys. Rev. Lett. 117, 136602 (2016).

[41] A. A. Burkov, Phys. Rev. B 96, 041110(R) (2017).

[42] M.-X. Deng, H.-J. Duan, W. Luo, W. Y. Deng, R.-Q. Wang, and L. Sheng, Phys. Rev. B 99, 165146 (2019).

[43] C. M. Wang, H.-Z. Lu, and S.-Q. Shen, Phys. Rev. Lett. 117, 077201 (2016).

[44] S. Hershfield and V. Ambegaokar, Phys. Rev. B 34, 2147 (1986).

[45] K.-S. Kim, H.-J. Kim, and M. Sasaki, Phys. Rev. B 89, 195137 (2014).

[46] S. Das Sarma, E. H. Hwang, and H. Min, Phys. Rev. B 91, 035201 (2015).

[47] K. Das and A. Agarwal, Phys. Rev. B 99, 085405 (2019).

[48] H. Geng, W.-Y. Deng, Y.-J. Ren, L. Sheng, and D.-Y. Xing, Chin. Phys. B 25, 097201 (2016).

[49] H. Geng, W. Luo, W. Y. Deng, L. Sheng, R. Shen, and D. Y. Xing, Sci. Rep. 7, 3755 (2017).

[50] A. A. Taskin, H. F. Legg, F. Yang, S. Sasaki, Y. Kanai, K. Matsumoto, A. Rosch, and Y. Ando, Nat. Commun. 8, 1340 (2017). 